\title{
REPORT OF A NEW CASE WITH PENTASOMY $X$ AND NOVEL CLINICAL FINDINGS
}

\author{
Demirhan $\mathrm{O}^{1, *}$, Tanriverdi $\mathrm{N}^{1}$, Yilmaz $\mathrm{MB}^{1}$, Kocaturk-Sel $\mathrm{S}^{1}$, \\ Inandiklioglu $\mathrm{N}^{1}$, Luleyap $\mathrm{U}^{1}$, Akbal E ${ }^{1}$, Comertpay $\mathrm{G}^{1}$, Tufan $\mathrm{T}^{1}$, Dur $\mathrm{O}^{2}$
}

\begin{abstract}
*Corresponding Author: Professor Dr. Osman Demirhan, Department of Medical Biology and Genetics, Faculty of Medicine, Çukurova University, 01330 Saricam, Adana, Turkey. Tel: +90-322-338-7140. Fax: +90-322338-6572. E-mail: osdemir@cu.edu.tr
\end{abstract}

\begin{abstract}
Pentasomy $\mathrm{X}$ is an extremely rare sex chromosome abnormality, a condition that only affects females, in which three more $\mathrm{X}$ chromosomes are added to the normally present two chromosomes in females. We investigated the novel clinical findings in a 1-year-old female baby with pentasomy $\mathrm{X}$, and determined the parental origins of the $\mathrm{X}$ chromosomes. Our case had thenar atrophy, postnatal growth deficiency, developmental delay, mongoloid slant, microcephaly, ear anomalies, micrognathia and congenital heart disease. A conventional cytogenetic technique was applied for the diagnosis of the polysomy X, and quantitative fluorescent polymerase chain reaction (QF-PCR) using 11 inherited short tandem repeat (STR) alleles specific to the chromosome $\mathrm{X}$ for the determination of parental origin of $\mathrm{X}$ chromosomes. A cytogenetic evaluation revealed that the karyotype of the infant was $49, \mathrm{XXXXX}$. Comparison of the infant's features with previously reported cases indicated a clinically recognizable specific pattern of malformations referred to as the pentasomy X syndrome. However, to the best of our know-ledge, this is the first report of thenar atrophy in a patient with $49, X X X X X$. The molecular analysis suggested that four $\mathrm{X}$ chromosomes of the infant originated from
\end{abstract}

\footnotetext{
${ }^{1}$ Department of Medical Biology and Genetics, Çukurova University, Faculty of Medicine, Adana, Turkey

${ }^{2}$ Department of Pediatrics, Çukurova University, Faculty of Medicine, Adana, Turkey
}

the mother as a result of the non disjunction events in meiosis I and meiosis II. We here state that the clinical manifestations seen in our case were consistent with those described previously in patients with pentasomy $\mathrm{X}$. The degree of early hypotonia constitutes an important early prognostic feature in this syndrome. The pathogenesis of pentasomy $\mathrm{X}$ is not clear at present, but it is thought to be caused by successive maternal non disjunctions.

Keywords: Clinical findings; parental origin; pentasomy X syndrome; quantitative fluorescentpolymerase chain reaction (QF-PCR); short tandem repeat (STR) markers; 49, XXXXX

\section{INTRODUCTION}

Pentasomy $\mathrm{X}$ is a very rare chromosome abnormality. The exact prevalence is unknown, and was first described by Kesaree and Wooley [1]. Approximately, 25 cases have so far been reported in the literature [2], although it is believed that there are probably many more who have never been diagnosed. Pentasomy $\mathrm{X}$ is associated with developmental delays, short stature, craniofacial anomalies (microcephaly, micrognathia, plagiocephaly, hypertelorism, up-slanting palpebral fissures, a flat nasal bridge and ear malformations), musculoskeletal abnormalities and cardiovascular malformations. The hands and feet are generally small with common findings of camptodactyly, clinodactyly and radioulnar synostosis. Immunoglobulin anomalies and increased susceptibility to infection have also 


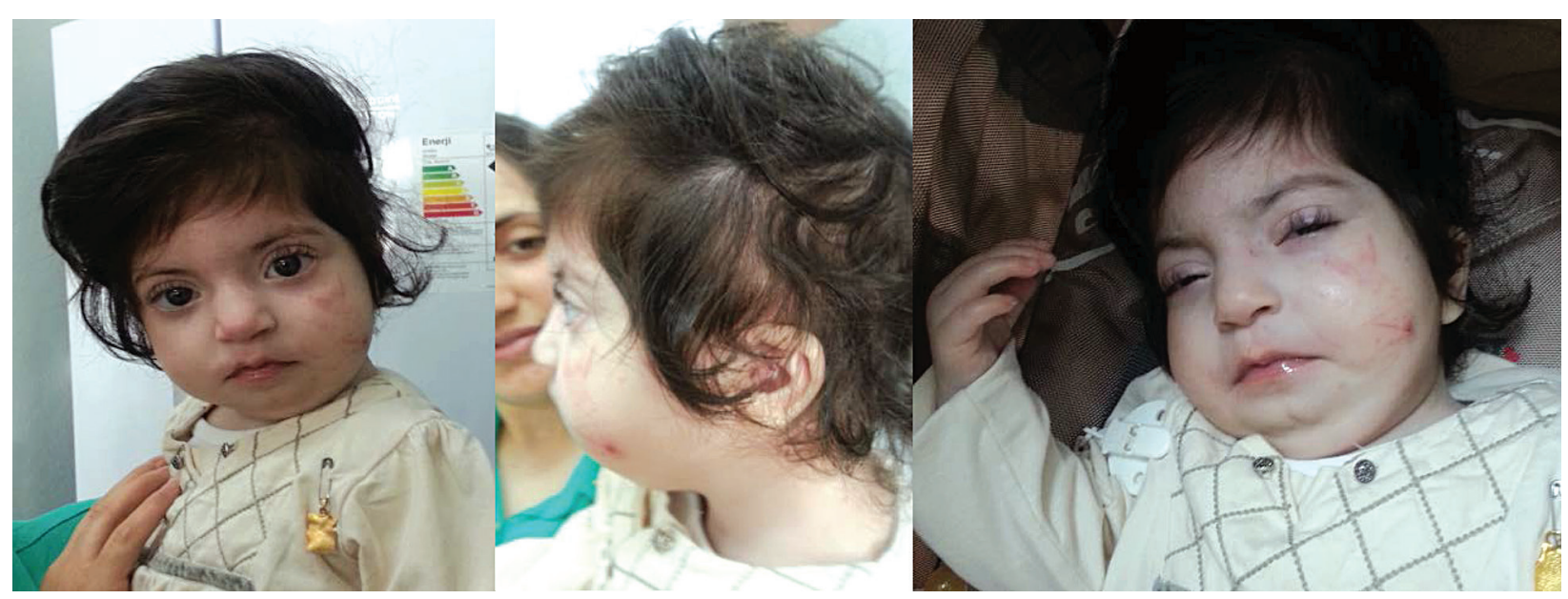

Figure 1. Front and side view of infant's face. Note mongoloid slant, epicanthus, microcephaly, low-set and upward ears and micrognathia

been reported in such cases. External genitalia are generally normal but gonadal dysfunction has been reported. The majority of polysomies are due to double non disjunction in oogenesis. The addition of more than one extra sex chromosome occurs rarely, and information in the literature is generally limited to isolated case reports. In this study, we present an infant with a 49,XXXXX chromosome and novel clinical findings of pentasomy $\mathrm{X}$. This case gave us the opportunity to study the effects of pentasomy X syndrome on phenotype.

\section{MATERIALS AND METHODS}

Case Report. She was born by Cesarean section during the 32 nd gestational week as a result of premature membrane rupture. The infant was a 1-yearold girl, the fourth child of healthy related parents (mother aged 31, father 45). Her birth weight was unknown. Her current weight was $8000 \mathrm{~g}$, length was $66 \mathrm{~cm}$ (25th percentile), and head circumference was $43 \mathrm{~cm}$ (75-90th percentile). Her siblings were healthy and developed normally. The infant was admitted to the inpatient clinic at the Department of Pediatrics, Çukurova University, Faculty of Medicine, Adana, Turkey, with a history of cough and fever for 20 days. Her physical examination revealed that she had a body temperature of $39^{\circ} \mathrm{C}$, a pulse rate of 128 beats $/ \mathrm{min}$. and a blood pressure of $100 / 60 \mathrm{~mm} / \mathrm{Hg}$. She had tachypnea and dyspnea with oxygen saturation of $88.0 \%$ in room air. Auscultation of chest revealed bilateral crepitations and the cardiovascular examination revealed 3/6 systolic murmur. Palpable hepatomegaly was present in the abdomen. The neurological examination was notable for diffuse hypotonia, tongue fasciculations and absent deep tendon reflexes. She also had oblique eye fissures, upward ears, thenar atrophy and normal external genitalia.

Initial laboratory tests showed a white blood cell (WBC) count of $16.0 \times 10^{9} / \mathrm{L}$ with $50.0 \%$ neutrophils, $42.0 \%$ lymphocytes, $4.0 \%$ monocytes, $4.0 \%$ eosinophils, a platelet count of $257.0 \times 10^{9} / \mathrm{L}$, a red blood cell (RBC) count of $3.7 \times 10^{12} / \mathrm{L}$, procalcitonin concentration of $24 \mathrm{ng} / \mathrm{ml}$, an alanine aminotransferase (ALT) level of $21 \mathrm{U} / \mathrm{L}$, a creatinine phospokinase (CPK) level of $36 \mathrm{U} / \mathrm{L}$ and a creatinine concentration of $0.41 \mathrm{mg} / \mathrm{dL}$. Her venereal diseases research laboratory (VDRL), cytomegalovirus, rubella, and toxoplasma tests were negative. The pediatric cardiology department of our hospital evaluated the patient. Transthoracic echocardiography revealed patent ductus arteriosus and a mild pulmonary hypertension so the patient was treated with captopril and furosemide drugs. The child also had pneumonia and was given antibiotics for 10 days. The pediatric neurology department evaluated the patient. According to her neurological findings and physical examination, spinal muscular atrophy was suspected, but genetic testing could not be performed to confirm the clinical diagnosis. The patient was discharged after 10 days of treatment. The child will regularly attend the pediatric cardiology and neurology polyclinic follow-up (Figure 1).

Cytogenetic Analysis. The diagnosis of the infant was made on the basis of a chromosomal analysis at the Department of Medical Biology and Genetics, 
Faculty of Medicine, Çukurova University, Adana, Turkey. Standard cytogenetic procedures were performed for the analysis of metaphase chromosomes from peripheral blood samples. Standard techniques for the cultivation of lymphocytes from peripheral blood were used and the preparations were treated with trypsin to obtain G-banding. The analyses were performed on $\geq 50$ cells. Karyotype analyses were as per International System for Human Cytogenetic Nomenclature (ISCN) (2005) standards.

Molecular Analysis. DNA was extracted from blood samples of the baby and her parents using InstaGene ${ }^{\mathrm{TM}}$ Matrix (Bio-Rad Laboratories, Hercules, CA, USA) according to the manufacturer's instructions. Quantitative fluorescent-polymerase chain reaction (QF-PCR) amplifications were performed employing Aneufast ${ }^{\mathrm{TM}}$ (Molgentix $\mathrm{SL}$, Barcelona, Spain) trisomy detection kit that includes fluorescently labeled primers for the 35 predefined short tandem repeat (STR) marker sites for chromosomes 13, 18, 21, X and Y, and primer pairs for amelogenin (AMXY) (specific to $X$ and $Y$ chromosomes) and sex-determining region $\mathrm{Y}$ (SRY) (specific to $\mathrm{Y}$ chromosome) regions. The kit also contains deoxynucleotide triphosphates (dNTPs) and Hot Start Taq DNA polymerase in an optimized reaction buffer. Two $\mu \mathrm{L}$ of the DNA (5-10 ng) and $3 \mu \mathrm{L}$ of PCR-grade water were added to $10 \mu \mathrm{L}$ of each of the master mixes. After the initial denaturation at $95{ }^{\circ} \mathrm{C}$ for 15 min., amplification was followed by 28 cycles at $95^{\circ} \mathrm{C}$ for 40 seconds, $58^{\circ} \mathrm{C}$ for 80 seconds and 72 ${ }^{\circ} \mathrm{C}$ for 40 seconds and final extension was $30 \mathrm{~min}$. at $60{ }^{\circ} \mathrm{C}$. The $\mathrm{QF}-\mathrm{PCR}$ products $(1.5 \mu \mathrm{L}$ from each mix) were collected in $20 \mu \mathrm{L}$ Hi-DiTM Formamide (Applied Biosystems, Foster City, CA, USA) containing $0.3 \mu \mathrm{L}$ of GeneScan ${ }^{\mathrm{TM}}-500 \mathrm{LIZ}^{\mathrm{TM}}$ (Applied

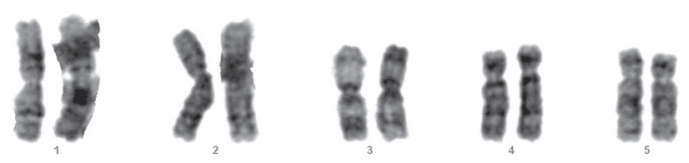 ถูก

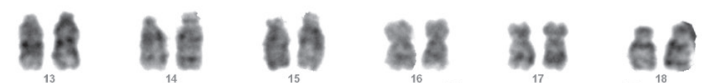

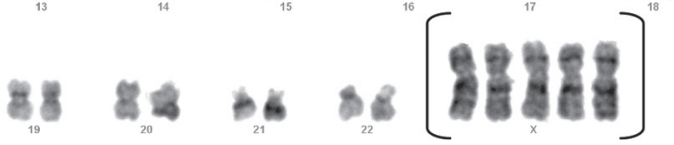

(A)

\section{III

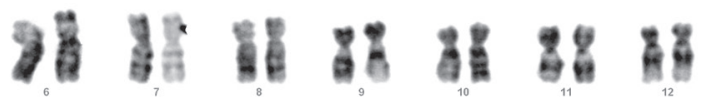

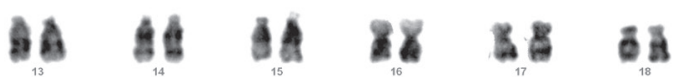

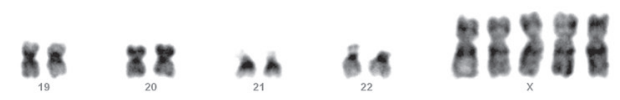

(C)

Figure 2. (A) G-band karyotype of the infant showing 49,XXXXX and pattern of the other chromosomal aberrations (B), del(13)(q22-p2) (C) chromatin break (3q), and (D) rosette formation.

\section{11 | $11 \mathrm{II}$ ใิ

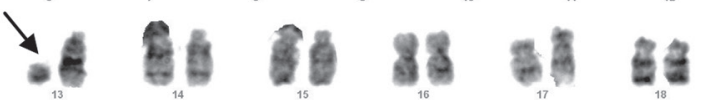

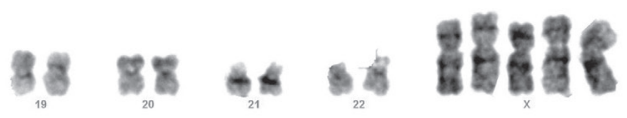

(B)

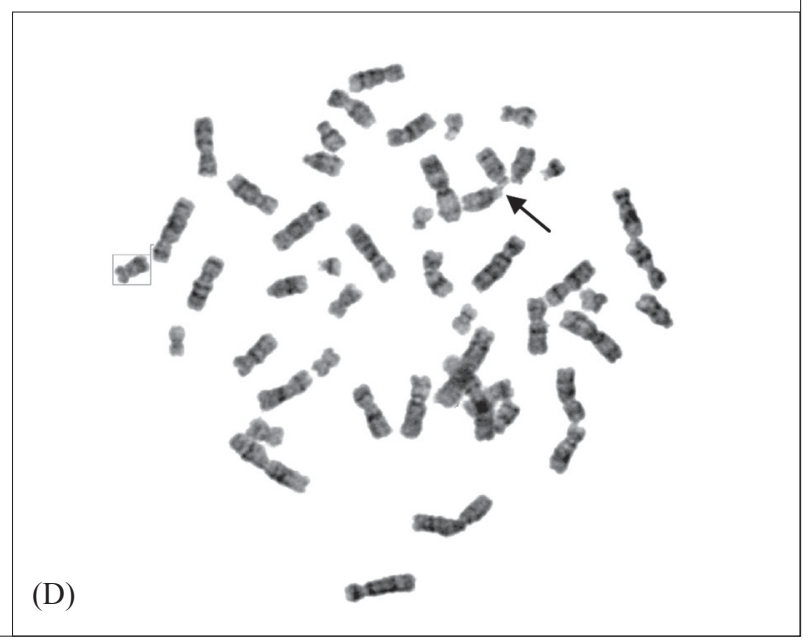




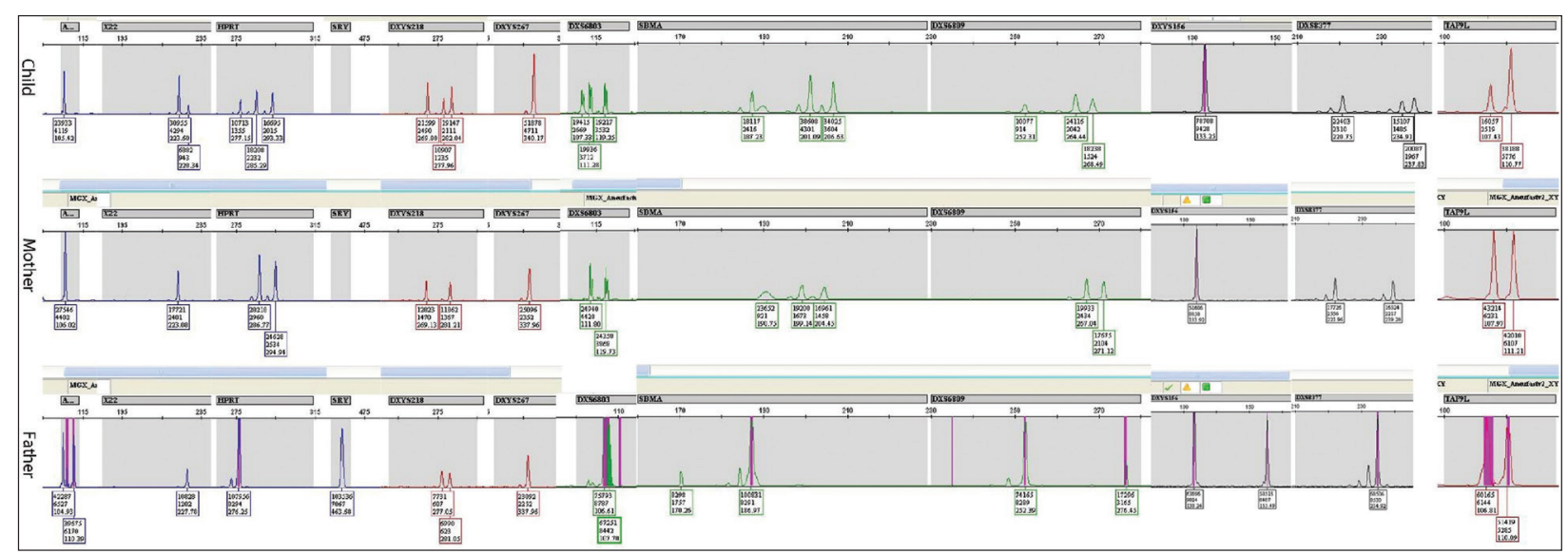

Figure 3. Electropherograms of the QF-PCR products of 11 STR markers on chromosome X (SBMA, DXS6803, DXS6809, DXS8377 and HPRT) and on X and Y chromosome STRs; AMXY, DXYS218, X22, DXYS267 and DXYS156 of the child, mother and father. The SRY is specific of chromosome Y. The TAF9L (on chromosomes 3 and $\mathrm{X}$ ) region was used for the determination and comparison of the number of the $\mathrm{X}$ and 3 chromosomes. The child's STR marker peak ratios were: 1:1 for AMXY, 4:1 for X22, 1:2:2 for HPRT, 2:1:2 for DXYS218, 1 for DXYS267, 1:2:2 for DXS6803, 1:2:2 for SBMA, 1:2:2 for DXS6809, 1 DXYS156, 2:1:2 for DXS8377, approximately 1:2.5 for TAF9L. The SRY is absent. The parents' STR markers have normal ratios.

Biosystems) size standard. After denaturation at 95 ${ }^{\circ} \mathrm{C}$ for 3 min., the mixture was allowed to cool down to $4{ }^{\circ} \mathrm{C}$ and then capillary electrophoresis was carried out on an ABI PRISM ${ }^{\text {TM }} 3130$ Genetic Analyzer using POP7 polymer (Applied Biosystems). Analysis of the results and calculation of the peak areas were performed using GeneMapper 4.0 software (Applied Biosystems).

Origin of the Aneuploidy. The parental origin of the aneuploidy was revealed by comparing of the STR alleles of the baby with that of her mother and father. The meiotic division errors that occurred either in meiosis I or meiosis II, was inferred on the basis of non reduction/reduction stage of the chromosome by comparing the proximal (peri-centromeric) markers. If parental heterozygosity was retained in the aneuploidic child, it is concluded that the error occurred during meiosis I, and if parental heterozygosity was reduced to homozygosity in the child, it is concluded that the error occurred during meiosis II or post-zygotic mitosis. Mitotic errors were distinguished from meiosis II by evaluating medial and distal markers. If the individual was reduced to homozygosity at all informative loci, including at least one each in proximal, medial, and distal portions of the chromosome, a post-zygotic origin was inferred. If the individual was not reduced to homozygosity at one or more loci, the error was assigned to meiosis II [3-6].

\section{RESULTS}

In this study, the mother had three previous pregnancies that resulted in the delivery of healthy children. In the present pregnancy, all of the infant cells analyzed had an abnormal karyotype of 49, XXXXX, and some cells have structural abnormalities such as del(13)(q22-p2), chromatin break (3q) and rosette formation (Figure 2). There was no evidence of mosaicism. The phenotypically normal siblings, mother and father all had normal karyotypes (46,XX or 46,XY) (Figure 3). In this study, 11 gender STR markers were amplified: chromosome X specific; SBMA, DXS6803, DXS6809, DXS8377 and HPRT; both chromosomes X- and Y-specific; AMXY, DXYS218, X22, DXYS267 and DXYS156; chromosome Y-specific, SRY and chromosomes 3and X-specific TAF9L. The TAF9L region was used for the determination of the numbers of chromosomes $\mathrm{X}$ and 3 to compare somatic $v S$. sex chromosomes. When the peak areas of AMXY and DXYS218 of the child, mother, and father were compared, it can be seen that there is an imbalance of the $\mathrm{X}$ chromosome in the child. This condition indicates the penta $\mathrm{X}$ condition of the child. As it was seen in Figure 3, all the chromosome X specific STR markers of the child inherited from the mother (origin of the aneuploidy is maternal) and heterozygosity was retained in all 
regions (proximal, medial and distal regions) of the four $\mathrm{X}$ chromosomes, this is the expected condition of non disjunction in both meiosis I and meiosis II. This showed that four of the $\mathrm{X}$ chromosomes of the child originated from the mother, and revealed a de novo tetrasomy of maternal chromosome $\mathrm{X}$. The other $\mathrm{X}$ chromosome originated from the father.

\section{DISCUSSION}

Pentasomy $\mathrm{X}$ is a rare chromosomal abnormality with an unknown prevalence, and has been associated with severe clinical conditions. Pentasomy X syndrome was first reported by Kesaree and Wooley in 1963 [1]. These patients usually have normal genitalia, but secondary sex characteristics can be incomplete. Pentasomy $\mathrm{X}$ syndrome has been associated with severe clinical conditions, presumably resulting from failure or disruption of X chromosome inactivation [7]. No adults with pentasomy $\mathrm{X}$ have been reported since the prognosis is universally poor. Here, we describe a case of 49,XXXXX karyotype in a 1-year-old girl. The principal features of the pentasomy X syndrome are set out in Table 1 and illustrated in Figure 1.

Pentasomy X chromosomes result sporadically from either meiotic non disjunction, where a chromosome fails to separate during the first or second division of gametogenesis, or from mitotic non disjunction in the developing zygote. If by chance the $\mathrm{X}$ chromosomes do not separate properly and they go on to the next cell division and again do not divide properly, when the sperm fertilizes the egg the fetus can then end up with four $X$ chromosomes and one X (49,XXXXX). Just as in our study, all the chromosome X-specific STR markers of the case were inherited from the mother (origin of the aneuploidy is maternal) and heterozygosity was retained in all regions (proximal, medial and distal regions) of the four chromosomes. This is the expected conditions of non disjunction in both meiosis I and meiosis II. Some researchers indicate that molecular genetic analyses using polymorphic DNA markers have enabled the study of non disjunction of fetal aneuploidy as well as determination of parental origin and cell stage errors at meiosis I or II $[3,5,6]$.

In the present study, the most important prognostic feature consists of extreme thenar atrophy, hypotonia, micrognathia and cardiovascular anoma- lies (Figure 1). Some of these clinical manifestations were consistent with those described previously in patients with pentasomy X, except for thenar atrophy. The hands and feet are generally small and camptodactyly, clinodactyly, and radioulnar synostosis are common findings in the pentasomy $\mathrm{X}$ syndrome. In addition to these hand findings, we report the first case with extreme thenar atrophy, which was not found in previous cases. A karyotype is therefore mandatory in infants with moderate-to-severe thenar atrophy, in order to exclude polyploidy $\mathrm{X}$ chromosome. Girls with a pentasomy X generally have mental retardation, mongoloid face, short neck, simian crease, clinodactyly of the fifth finger and congenital heart disease [7]. Because most patients with pentasomy X syndrome (including ours) have developmental delay, postnatal growth deficiency, epicanthus and a mongoloid slant of facial anomalies and hypotonia, similar to the abnormalities seen in Down's syndrome, occasional diagnostic problems have also been reported [8] (Figure 1). Because patients can be misdiagnosed as having Down's syndrome, the correct diagnosis requires cytogenetic analysis.

The patients with pentasomy X syndrome have a poor prognosis due to severe cardiac failure and infectious diseases [2,9-12]. Immunoglobulin anomalies and an increased susceptibility to infection have also previously been reported in patients with pentasomy $\mathrm{X}$. Immunoglobulin values in our case had not been tested. However, history of a greatly increased incidence of recurrent infections and fever was found in our case, whereas Boeck et al. [13] described their patient as having a lifelong history of eczema, recurrent pneumonia and staphylococcal abscess. Pneumonia development from early infancy, as seen in our case, has also been described in this syndrome. The normal external genitalia seen in our case have been reported in previous patients, although gonadal dysfunction has been seen in many cases, including a postmortem case [1,14-16]. These findings suggest that despite the normal appearance of the external genitalia, there is an underlying gonadal dysfunction in patients with pentasomy $\mathrm{X}$ syndrome. Because our case was an infant, her sexual development and bone maturation remain to be assessed. Growth deficiency was evident in the majority of affected children at the time of birth. Birth weight of our case was unknown. However, she was born by Cesarean section during the $32 \mathrm{nd}$ 
Table 1. Pattern of malformations in 20 patients with the pentasomy X syndrome (number of patients who had a specific phenotypic feature/number of evaluated patients for a specific feature) versus phenotypic features observed in our proband $[1,8,9,11,12,14,16]$.

\begin{tabular}{|c|c|c|c|c|c|}
\hline $\begin{array}{l}\text { Phenotypic Features } \\
\text { in the Literature }\end{array}$ & $\begin{array}{l}\text { Observed/ } \\
\text { Evaluated }\end{array}$ & $\begin{array}{c}\text { Our } \\
\text { Proband }\end{array}$ & $\begin{array}{l}\text { Phenotypic Features } \\
\text { in the Literature }\end{array}$ & $\begin{array}{l}\text { Observed/ } \\
\text { Evaluated }\end{array}$ & $\begin{array}{c}\text { Our } \\
\text { Proband }\end{array}$ \\
\hline Performance & & & Limbs & & \\
\hline postnatal growth deficiency & $9 / 17$ & {$[+]$} & elbow defects & $7 / 20$ & {$[-]$} \\
\hline developmental delay & $10 / 17$ & {$[+]$} & micromelia & $5 / 20$ & {$[-]$} \\
\hline mental retardation & $3 / 6$ & $?$ & fifth finger clinodactyly & $10 / 20$ & {$[-]$} \\
\hline Facial Features & & & low dermal ridge count & $9 / 15$ & {$[-]$} \\
\hline mongoloid slant & $2 / 7$ & {$[+]$} & positional foot deformities & $410 / 19$ & {$[-]$} \\
\hline midfacial hypoplasia & $2 / 7$ & {$[-]$} & hyperextensible elbow joints & $2 / 7$ & {$[-]$} \\
\hline ocular hypertelorism & $13 / 20$ & {$[-]$} & valgus of the feet & $1 / 6$ & {$[-]$} \\
\hline microcephaly & $8 / 17$ & {$[+]$} & small hands/feet & $1 / 6$ & {$[-]$} \\
\hline epicanthal folds & $1 / 19$ & {$[-]$} & Congenital Heart Disease & $9 / 20$ & {$[+]$} \\
\hline upward slanting palpebral fissures & $8 / 19$ & {$[-]$} & Skeletal Anomalies & & \\
\hline hypertelorism & $2 / 6$ & {$[-]$} & proximal radioulnar synostosis & $2 / 6$ & {$[-]$} \\
\hline ear anomalies & $11 / 20$ & {$[+]$} & hand arachnodactyly & $1 / 6$ & {$[-]$} \\
\hline depressed nasal bridge & $1 / 6$ & {$[-]$} & hyperlaxity of joints & $2 / 7$ & {$[-]$} \\
\hline flat nasal bridge & $1 / 6$ & {$[-]$} & thenar atrophy & $1 / 6$ & {$[+]^{\mathrm{a}}$} \\
\hline flat upturned nose & $1 / 6$ & {$[-]$} & Neurological Anomalies & & \\
\hline micrognathia & $2 / 6$ & {$[+]$} & hypotonia & $4 / 7$ & {$[+]$} \\
\hline retrognathia & $3 / 7$ & {$[-]$} & & & \\
\hline cleft palate & $1 / 6$ & {$[-]$} & & & \\
\hline short neck & $9 / 20$ & {$[-]$} & & & \\
\hline dental anomalies & $7 / 18$ & {$[-]$} & & & \\
\hline
\end{tabular}

$[+]$ : present, $[-]$ : absent.

${ }^{a}$ Novel feature observed in our proband.

gestational week as a result of premature membrane rupture, and had postnatal growth deficiency and developmental delay; these anomalies have also been described in this syndrome. Her intellectual functions had not been formally tested, but were suspected to be at low-average.

Patients with pentasomy X syndrome generally have congenital heart defects; usually patent ductus arteriosus or a ventricular septum defect $[1,8,9,11,12,14,16]$. Just as documented, cardiac defects were present in nine $(45.0 \%)$ of 20 children with 49,XXXXX (Table 1). Our case had a surgical operation for cardiac murmurs and patent ductus arteriosus. Reports in the medical literature agree that around $50.0 \%$ of girls are born with a heart condition. However, the medical literature includes girls with an innocent heart murmur. The most common defects are holes between the lower chambers of the heart and a condition called persistent ductus arteriosus that is left-over from the way the circulation operates during fetal life. Some cases are born with a more complex heart condition and will need a surgical operation. Three of those children developed congestive heart failure within the first year of life and required surgical ligation of the ductus. A karyotype is therefore mandatory in female newborns with moderate-to-severe hypotonia (including our case), in order to exclude polyploid mosaicism of the $\mathrm{X}$ 
chromosome [17]. Genetic counseling is indicated during subsequent pregnancies. Parents must realize that, apart from somatic areas being affected (facial dysmorphism, skeletal, cardiovascular and gonadal systems), all major areas of development will be affected by the addition of extra X chromosomes. However, an overall prognosis seems impossible due to a substantial variability within all polysomy $\mathrm{X}$ groups. In addition, the other structural abnormalities, del(13)(q22-p2) and chtbr(3q), were observed in two cells of our case (Figure 2). This loss of chromosome and chromatid break may affect susceptibility to the progression of the disease.

In conclusion, the clinical manifestations observed in our case were consistent with those described previously in patients with pentasomy X. To the best of our knowledge, we are the first to report thenar atrophy in a case with $49, \mathrm{XXXXX}$. Chromosome karyotyping is generally required in the presence of facial dysmorphism or other somatic abnormalities, to exclude underlying sex chromosomal aneuploidy disorder, such as the 49, XXXXX syndrome. Additional studies about polyploidies are needed to determine the nature of the errors in such cases. Evaluation of exceptional instances of segregation failure may be useful in improving our understanding of the general mechanisms of non disjunction. Molecular techniques could be useful for the pre and postnatal diagnosis of the common aneuploidies and determining its parental origin. These kinds of studies will improve our knowledge about the mechanisms of aneuploidies, and enable appropriate and prompt genetic counseling.

Declaration of Interest. The authors report no conflicts of interest. The authors alone are responsible for the content and writing of this article.

\section{REFERENCES}

1. Kesaree N, Wolley PV. A phenotypic female with 49 chromosomes, presumably XXXXX. J Pediatr. 1963; 63(1): 1099-1103.

2. Linden MG, Bender BG: Robinson A sex chromosome tetrasomy and pentasomy. Pediatrics 1995; 96(4): 672-681.

3. Lamb NE, Freeman SB, Savage-Austin A, Pettay D, Taft L, Hersey J, et al. Susceptible chiasmate configurations on chromosome 21 predispose to non-disjunction in both maternal meiosis I and meiosis II. Nat Genet. 1996; 14(4): 400-405.

4. Nicolaidis P, Petersen MB. Origin and mechanisms of nondisjunction in human autosomal trisomies. Hum Reprod. 1998; 13(2): 313-319.

5. Robinson WP, Bernasconi-Quadroni F, Lau A, McFadden DE. Origin of trisomy: Effect of ascertainment. Am J Med Genet. 1999; 84(1): 34-42.

6. Diego-Alvarez D, Ramos-Corrales C, GarciaHoyos M, Bustamante-Aragones A, Cantalapiedre $\mathrm{D}$, Diaz-Recasens J, et al: Double trisomy in spontaneous miscarriages: Cytogenetic and molecular approach. Hum Reprod. 2006; 21(4): 958-966.

7. Moraes LM, Cardoso LC, Moura VL, Moreira MA, Menezes AN, Llerena JC Jr, et al. Detailed analysis of $\mathrm{X}$ chromosome inactivation in a 49, XXXXX pentasomy. Mol Cytogenet. 2009; 2(1): 20-33.

8. Cho YG, Kim DS, Lee HS, Cho SC, Choi SI. A case of 49, XXXXX in which the extra X chromosomes were maternal in origin. J Clin Pathol. 2004; 57(1): 1004-1006.

9. Kassai R, Hamada I, Furuta H, Cho K, Abe K, Deng $\mathrm{H}-\mathrm{X}$, et al. Penta X syndrome: A case report with review of the literature. Am J Med Genet. 1991; 40(1): 51-56.

10. Zhang R, Pan N, Li X, Wang XQ, Wu M. A case of 49,XXXXX syndrome. Chin Med J. 1982; 95(12): 891-894.

11. Peet J, Weaver DD, Vance GH. 49,XXXXY: A distinct phenotype. Three new cases and review. Med Genet. 1998; 35(5): 420-424.

12. Monheit A, Francke U, Saunders B, Jones KL. The penta-X syndrome. J Med Genet. 1980; 17(5): 392-396.

13. Boeck A, Gfatter R, Braun F, Fritz B. Pentasomy $\mathrm{X}$ and hyper IgE syndrome: Coexistence of two distinct genetic disorders. Eur J Pediatr. 1999; 158(9): 723-726.

14. Sergovich F, Uilenberg C, Pozsonyi J. The 49, XXXXX chromosome constitution: Similarities to the $49, \mathrm{XXXXY}$ condition. J Pediatr. 1971; 78(2): 285-290.

15. Toussi T, Halal F, Lesage R, Delorme F. Bergeron A. Renal hypodysplasia and unilateral ovarian agenesis in the penta-X syndrome. Am J Med Genet. 1980; 6(2): 153-162. 
16. Farge P, Dallaire L, Albert G, Melanĉon SB, Potter M, Leboeuf G. Oral and dental development in X chromosome aneuploidy. Clin Genet. 1985; 27(2): 122-126.
17. Schoubben E, Decaestecker K, Quaegebeur K, Danneels L, Mortier G, Cornette L. Tetrasomy and pentasomy of the $\mathrm{X}$ chromosome. Eur $\mathrm{J}$ Pediatr. 2011; 170(1): 1325-1327. 